\title{
Silencing of the glypican-3 gene affects the biological behavior of human hepatocellular carcinoma cells
}

\author{
XIN-HUI QI, DI WU, HUI-XIA CUI, NAN MA, JIA SU, YU-TONG WANG and YOU-HONG JIANG \\ Cancer Research Institute, The First Affiliated Hospital of China Medical University, Shenyang, Liaoning 110001, P.R. China
}

Received November 13, 2013; Accepted June 5, 2014

DOI: $10.3892 / \mathrm{mmr} .2014 .2600$

\begin{abstract}
Hepatocellular carcinoma (HCC) is one of the leading causes of cancer death in the world. The gene glypican-3 $(G P C 3)$ is reported to be a potential therapeutic target for HCC. In this study, we use RNA interference with lentiviral vectors to explore the effect of GPC3 silencing on the biological behavior of HCC cells and the potential role of the GPC3 protein in the activation of epithelial-mesenchymal transition (EMT), which relates to HCC cell invasion and migration. Our data suggest that GPC3 silencing leads to a decrease in $\mathrm{HCC}$ cell proliferation and to an increase in apoptosis. We demonstrated that GPC3 silencing regulates cell invasion and migration, most probably through the activation of the EMT cellular program. In conclusion, GPC3 is associated with the HCC cell biological behavior, while the relationship between GPC3 and EMT in tumorigenesis of HCC deserves future investigation.
\end{abstract}

\section{Introduction}

Hepatocellular carcinoma (HCC) is one of the most common malignant tumors in the world (1). It is the third common cause of cancer mortality worldwide, especially in the developing countries. HCC patients show a poor 5-year survival rate, since most of them are diagnosed at a late stage (2). HCC is associated with high potential for vascular invasion, metastasis, and recurrence even after surgical resection, leading to poor prognosis (3). HCC is a multi-step processes including numerous gene changes that associate with cell proliferation, apoptosis, invasion and metastasis. Thus, there is an urgent demand for early diagnosis and the development of novel molecular targets for HCC. In recent years, as the development of molecular-based therapies has emerged, increasing research focus has been placed on the identification of

Correspondence to: Dr You-Hong Jiang, Cancer Research Institute, The First Affiliated Hospital of China Medical University, 155 Nanjing North Street, Heping, Shenyang, Liaoning 110001, P.R. China

E-mail: jiangyouhong@mail.cmu.edu.cn

Key words: hepatocellular carcinoma, glypican-3, RNA interference, epithelial-mesenchymal transition, cell invasion, cell migration, Wnt/ $/$-catenin signaling pathway biomarkers, the expression of which is altered during the development of HCC (4-6). Numerous studies have shown that biomarkers may be promising molecular targets for the treatment of HCC (7-9).

The protein glypican-3 (GPC3) is a valuable diagnostic marker and a potential therapeutic target for HCC (10). It has been reported that this protein is almost not expressed in healthy liver or non-tumor tissues, while strong positive staining was observed for GPC3 at carcinoma sites (11-14). Furthermore, GPC3 is highly expressed in melanoma, ovarian clear-cell carcinomas, yolk sac tumors, neuroblastoma, hepatoblastoma, Wilms' tumor cells, and other tumors (15-19). By contrast, the gene appears silenced in breast cancer, mesothelioma, epithelial ovarian cancer and lung adenocarcinoma (20-22). Based on these reports, it was proposed that GPC3 is highly and specifically expressed in $\mathrm{HCC}$, and its overexpression appears not to inhibit HCC, but rather, promote it (23). GPC3 exerts positive or negative effects on cell growth, depending on the cell type $(24,25)$. The gene was first identified by Filmus et al (26) in 1988, and was named by Pilia et al (27) in 1996. GPC3 is located on the human $\mathrm{X}$ chromosome (Xq26) and encodes a 70-kDa core protein with 580 amino acids. It is a member of the glypican family, which has a basic structure consisting of a core protein, a heparan sulfate chain, and a glycosylphosphatidylinositol (GPI) anchor via which it attaches to the cell membrane $(28,29)$. As suggested by a previous study, GPC3 regulates cell morphology, adhesion, apoptosis, proliferation, migration, survival and differentiation by receiving signals from receptors on the cell surface (18). The protein can crosstalk with a number of signaling pathways during the oncogenesis of HCC. Although numerous biochemical and genetic studies have been performed to elucidate the role of GPC3 in modulating cell biological behavior $(25,30)$, the molecular mechanisms underlying GPC3-mediated invasion and migration remain elusive. The role of GPC3 in tumorigensesis deserves further investigation.

The epithelial-mesenchymal transition (EMT) is a key event in the tumor invasion process, whereby epithelial cell layers loose polarity and cell-cell contacts and undergo dramatic remodeling of the cytoskeleton (31). It is a process involving dissociation of adherens junctions and changes in cell morphology.

The present study focused on the role of GPC3 in the oncogenesis of HCC and the potential molecular events promoting 
cell invasion induced by GPC3. We silenced the GPC3 gene in HepG2 cells with an RNA interference method using lentiviral vectors, thereby reducing its expression at both the protein and mRNA levels. In addition, we detected the expression of EMT-related proteins by western blot analysis in order to investigate the progress of EMT in the GPC3-silenced cells. Our results reveal a potential link between GPC3-mediated invasion and the EMT process. Taken together, our study provides evidence that GPC3 may be an effective therapeutic target for treatment of HCC and may play an important role in gene therapy of HCC.

\section{Materials and methods}

Cell lines and culture conditions. The human HCC cell line HepG2 and the 293T cell line were purchased from the Cell Collection of the Chinese Academy of Sciences (Shanghai, China). Both cell lines were cultured in Dullbecco's modified Eagle's medium (DMEM), supplemented with $10 \%$ fetal bovine serum (FBS) (both HyClone ${ }^{\circledR}$, commercialized by Thermo Fisher Scientific, Waltham, MA, USA) at $37^{\circ} \mathrm{C}$ in a humidified atmosphere of $5 \% \mathrm{CO}_{2}$ and $95 \%$ air.

Construction of a recombinant lentiviral vector targeting GPC3. Small interfering RNA (siRNA) sequences targeting the human GPC3 gene (GenBank accession no., NP_004475) were designed following standard principles for the design of RNA interference sequences using Designer 3.0 (GenePharma, Shanghai, China). We selected the sequence 5'-GGCTCTGAATCTTGGAATT-3' in order to silence the expression of GPC3. The scrambled sequence 5'-TTCTCCGAACGTGTCACGT-3' was used as a negative control. The lentivirus PGLV3-green fluorescent protein (GFP) vector was purchased from GenePharma (Shanghai, China). Short hairpin RNAs (shRNAs) were generated based on the above siRNA sequences and were cloned into the PGLV3-GFP vector. The resulting plasmids were transfected into $293 \mathrm{~T}$ cells using Invitrogen ${ }^{\mathrm{TM}}$ Lipofectamine 2000 (Thermo Fisher Scientific) according to the manufacturer's instructions. At 48 and $72 \mathrm{~h}$ following the transfection, GFP expression was detected under a fluorescent microscope (Olympus IX71, Olympus, Tokyo, Japan; Apogee Alta U2 Cooled Camera, Sartorius Instrument System, Beijng, China) to determine the vector titer. Then, viral products were aliquoted and stored at $-80^{\circ} \mathrm{C}$ in DMEM containing $2.5 \%$ FBS. Finally, HepG2 cells were transfected with the appropriate titer, which was selected based on the transfection efficiency, measured through the expression of GFP under the fluorescent microscope.

Reverse transcription-quantitative polymerase chain reaction $(R T-q P C R)$. RT-qPCR was performed to determine the mRNA level of GPC3 in HCC following transfection with the lentiviral vector. Total RNA was isolated using the TRIzol reagent (Takara Bio Inc., Dalian, China) and reverse transcribed to cDNA using the RT Master Mix (Takara). The resulting cDNA was then used for measurement of RNA abundance by qPCR. Amplification was performed using the SYBR-Green PCR Master mix (Takara), with $100 \mathrm{ng}$ of cDNA in $20 \mu \mathrm{l}$ of the final reaction mixture. We used the following primer sequences $\left(5^{\prime}-3^{\prime}\right)$ : forward, GATGAGTGCATTGGAGGCTCTG, and reverse, ATGAACGTTCCCGAGGTTGTG. The cycling conditions were the following: one cycle at $95^{\circ} \mathrm{C}$ for $30 \mathrm{sec}, 40$ cycles at $95^{\circ} \mathrm{C}$ for $5 \mathrm{sec}$, and $60^{\circ} \mathrm{C}$ for $30 \mathrm{sec}$, and one cycle at $95^{\circ} \mathrm{C}$ for $15 \mathrm{sec}, 60^{\circ} \mathrm{C}$ for $30 \mathrm{sec}$, and $95^{\circ} \mathrm{C}$ for $15 \mathrm{sec}$. Three independent experiments were performed for each sample. The data were analyzed by comparing the $2^{-\Delta \Delta \mathrm{Ct}}$ values (32).

Annexin V-phycoerythrin (PE)/7-aminoactinomycin D (7-A $A D)$ apoptosis assay. To study the effect of GPC3 silencing on HepG2 cell apoptosis, cells were harvested after a $72 \mathrm{~h}$ transfection by brief trypsinization and were washed in phosphate-buffered saline (PBS) twice. Then, we used the Annexin V-PE/7-AAD staining kit (KeyGen Biotech, Nanjing, China) to detect the apoptotic rate of HepG2 cells. Cells were suspended in $500 \mu \mathrm{l}$ of binding buffer and incubated at room temperature in the dark for $15 \mathrm{~min}$ after labeling with $5 \mu \mathrm{l}$ of Annexin V/7-AAD and $1 \mu \mathrm{l}$ of Annexin V-PE. The stained cells were then analyzed by flow cytometry (FACSCalibur; Becton-Dickinson, Franklin Lakes, NJ, USA) and CellQuest software (BD Biosciences, Franklin Lakes, NJ, USA). The experiments were performed in triplicate.

Cell proliferation assay. To further study the effect of GPC3 silencing on cell proliferation, we used the cell counting kit- 8 (cck-8) assay (Beyotime Institute of Biotechnology, Shanghai, China) following the manufacturer's instructions. Briefly, $1 \times 10^{4} /$ well cells were seeded onto 96 -well plates and incubated at $37^{\circ} \mathrm{C}$ for 24,48 and $72 \mathrm{~h}$. Following cell attachment, $10 \mu \mathrm{l}$ CCK-8 were added to each well, and cells were incubated at $37^{\circ} \mathrm{C}$ for $2 \mathrm{~h}$. The optical density (OD) was measured at $450 \mathrm{~nm}$ using a microplate reader (RT-6000; Rayto Life and Analytical Sciences Co, Ltd., Shenzhen, China). Three independent experiments were performed.

Transwell Matrigel invasion assay. To evaluate the effect of silencing of GPC3 on HepG2 cell invasion, we used 24-well Transwell chambers with $8.0 \mu \mathrm{m}$ pore membranes (Corning, Inc., Corning, NY, USA). Following transfection, cells were seeded into the upper chamber at a density of $2 \times 10^{4}$ in $200 \mu \mathrm{l}$ serum-free medium. The lower chamber contained $600 \mu \mathrm{l}$ medium supplemented with $10 \%$ FBS as a chemoattractant. After incubation for $48 \mathrm{~h}$, the remaining cells on the upper surface of the filters were removed with cotton swabs, and migrating cells were stained with crystal violet and observed under a confocal microscope (DM77300B; Leica Microsystems, Mannheim, Germany). Invading cells were quantified by counting cells in 10 random fields at x100 magnification. Three independent experiments were performed.

Wound healing assay. To determine the effect of GPC3 silencing on HepG2 cell migration, a wound healing assay was performed. Cells $\left(6 \times 10^{5}\right)$ were seeded onto 6-well plates at $80 \%$ confluence. The cells were treated with serum-free medium and then wounded with a pipette tip of $10 \mu \mathrm{l}$. After washing with PBS three times, images were acquired at 0 and 24 h of incubation after wounding, using a microscope (DM77300B; Leica Microsystems). Images of the same area 


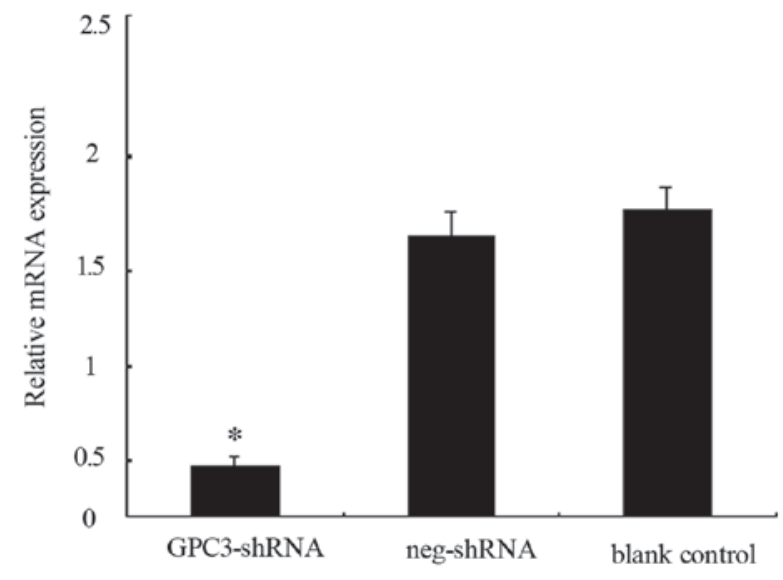

Figure 1. Effect of glypican-3 (GPC3) silencing on the mRNA level of the gene, as assessed by reverse transcription-quantitative polymerase chain reaction analysis. The mRNA level of $G P C 3$, expressed relative to that of GAPDH, is reduced in GPC3-shRNA-transfected HepG2 cells compared to the negative control scrambled shRNA-transfected and the blank control (non-transfected) cells. ${ }^{*} \mathrm{P}<0.05$ vs. control groups (three independent experiments, each with three mRNA samples in duplicate).

were acquired to determine wound coverage due to cellular motility. Data were quantified by measuring the scratch area of every field of vision. The assay was performed in triplicate.

Western blot assay. Total cellular protein was extracted following transfection, using the radio-immunoprecipitation assay (RIPA; Beyotime Institute of Biotechnology, Shanghai, China). The protein concentrations were determined by the bicinchoninic acid assay (BCA; KeyGen Biotech Co, Ltd., Nanjing China). SDS-PAGE was performed on $10 \%$ glycine gels to separate the proteins, which were then transferred onto polyvinylidene difluoride (PVDF) membranes. The PVDF membranes were blocked with 5\% non-fat milk in Tris-buffered saline and Tween-20 (TBST) buffer for $1 \mathrm{~h}$, then incubated with rabbit polyclonal anti-human anti-GPC3 (ProteinTech Group, Hubei, China) and anti- $\beta$-actin (Santa Cruz Biotechnology, Inc., Santa Cruz, CA, USA) primary antibodies overnight at $4^{\circ} \mathrm{C}$, followed by incubation with a horseradish peroxidase-conjugated anti-rabbit IgG (Santa Cruz Biotechnology, Inc.) as the secondary antibody. Protein bands were detected using the enhanced chemiluminescence (ECL) kit (CoWin Biotech, Beijing, China). To further investigate the mechanism underlying the changes in biological functions induced by GPC3 silencing, we also assessed the expression of proteins using mouse monoclonal anti-human anti-matrix metalloproteinase-2 (MMP-2), mouse monoclonal anti-human anti-MMP-9, mouse monoclonal anti-human anti$\beta$-catenin, rabbit polyclonal anti-human anti-E-cadherin, mouse monoclonal anti-human anti-Slug and rabbit polyclonal antihuman anti-Snail using the same procedure. The antibodies were purchased from Santa Cruz Biotechnology, Inc. The intensity of the bands on the gels was quantified using Image $J$ software (National Institutes of Health, Bethesda, MA, USA) and the experiments were repeated over five times.

Statistical analysis. Data were expressed as mean \pm standard deviation (SD) and subjected to one-way analysis of variance using the SPSS 17.0 software (SPSS Inc., Chicago, IL, USA). Differences between groups were examined by

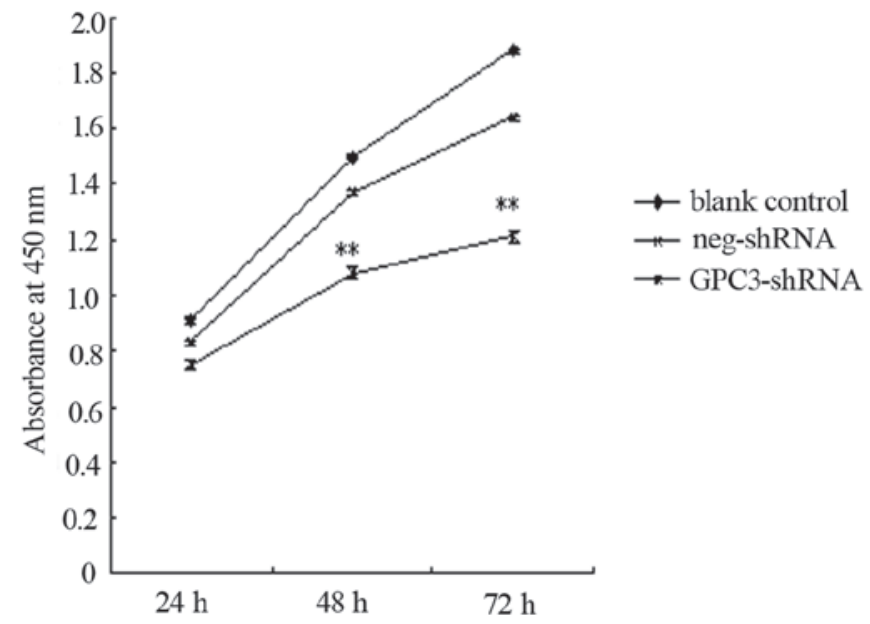

Figure 2. Effect of glypican-3 (GPC3) silencing on HepG2 cell proliferation. Following transfection with the GPC3-shRNA and the scrambled shRNA (neg-shRNA), HepG2 cells (1x10\%/well) were seeded into 96-well plates and incubated in culture medium for 24, 48 and $72 \mathrm{~h}$. Absorbance was measured $2 \mathrm{~h}$ after addition of cell-counting kit $8 .{ }^{* *} \mathrm{P}<0.05$ vs. control groups. The experiments were repeated three times.

Student's t-tests. Statistical significance was accepted at a p-value $<0.05$.

\section{Results}

The expression of GPC3 is reduced upon GPC3-shRNA transfection. Transfection with the GPC3-shRNA decreased the expression of GPC3 in HepG2 cells at both the mRNA and protein levels. RT-qPCR and western blot analysis were used to evaluate the differences between the transfected group and the blank control (non-transfected cells). The results showed that the expression level of the GPC 3 mRNA (Fig. 1) and protein (Fig. 5, upper right panel) were significantly reduced following transfection of HepG2 cells. These results indicated that the recombinant lentiviral vector for GPC3 silencing was successfully constructed.

GPC3 gene silencing decreases cell proliferation in HepG2 cells. The CCK-8 assay, a sensitive and specific method for the assessment of cell proliferation, was carried out to analyze the effects of GPC3 silencing on HepG2 cell proliferation. Following transfection with the GPC3-shRNA, proliferation was significantly decreased compared to the control group (Fig. 2). This result indicated that silencing of GPC3 may decrease the growth and survival of the HepG2 cells.

Silencing of the GPC3 gene increases the apoptotic rate of HepG2 cells. Resistance to apoptosis is a characteristic feature of tumor cells, and we thus investigated whether GPC3 is associated with cell apoptosis. We used the Annexin V PE/7-AAD assay to analyze the effect of GPC3 silencing on cell apoptosis. The data shown in Fig. 3 reveal that, compared to the control group, the apoptotic rate of HepG2 cells that were GPC3-silenced was notably increased $(\mathrm{P}<0.05)$. We conclude that GPC3 may affect cell apoptosis, since the inhibition of its expression markedly increases HepG2 cell apoptosis. 


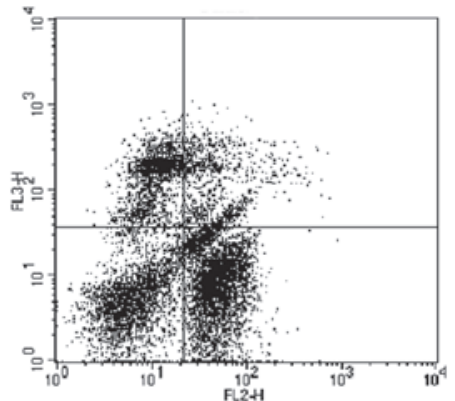

GPC3-shRNA

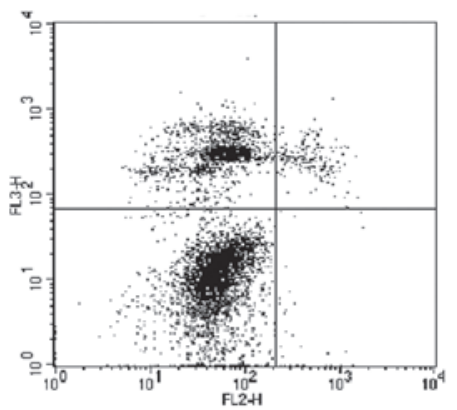

blank control
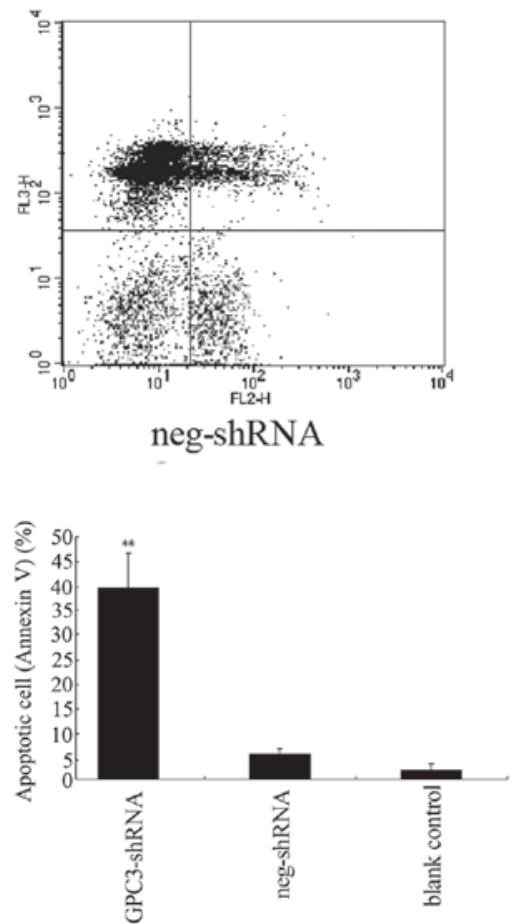

Figure 3. Effect of glypican-3 (GPC3) silencing on cell apoptosis. Adherent cells (1x105/ml) transfected with GPC3-shRNA and scrambled shRNA (neg-shRNA control) were serum-starved, double-stained with Annexin V/phycoerythrin (PE) and Annexin V/7-aminoactinomycin D (7-AAD), and harvested after 72 h of transfection. The percentage of apoptotic cells was quantified by flow cytometry. ${ }^{* *} \mathrm{P}<0.05$ vs. control groups. The experiments were repeated three times.

A

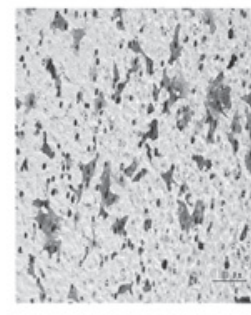

GPC3-shRNA
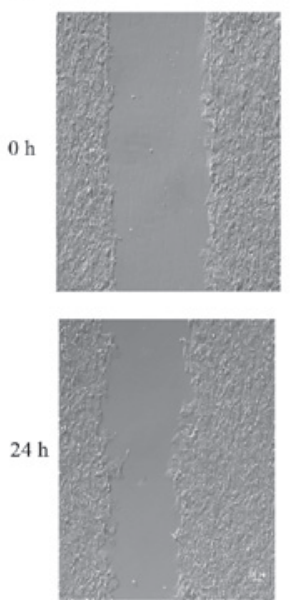

blank control

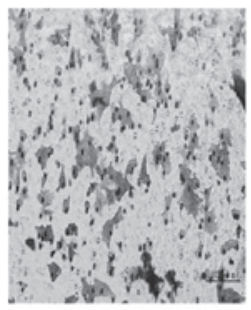

neg-shRNA
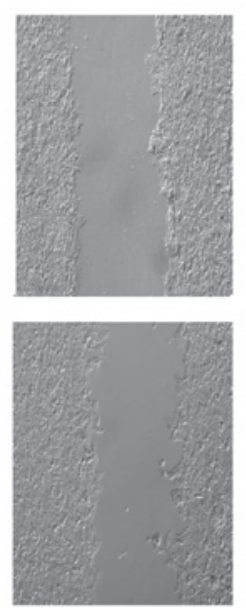

neg-shRNA

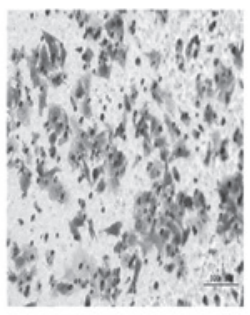

blank control
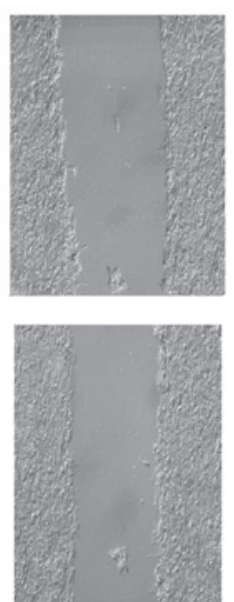

GPC3-shRNA
B

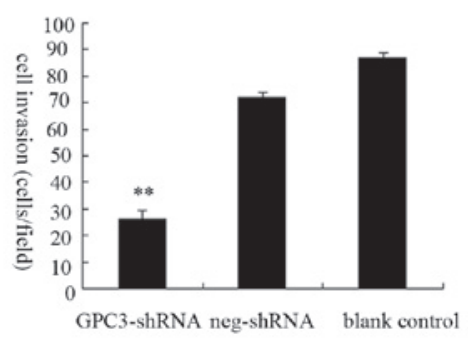

Figure 4. Silencing of glypican-3 (GPC3) reduces the migratory and invasive ability of HepG2 cells. (A) The upper wells of the Transwell chambers were coated with Matrigel and seeded with HepG2 cells transfected with GPC3-shRNA and negative-shRNA, and supplemented with serum-free medium. Cell invasion was assayed after $48 \mathrm{~h}$. After removal of cells from the upper surface of the chambers, the membranes were stained with crystal violet. (B) Invading cells were quantified by counting cells in 10 random fields at $\mathrm{x} 100$ magnification. ${ }^{* *} \mathrm{P}<0.05$ vs. control groups. (C) Images of cells at $0 \mathrm{~h}$ and $24 \mathrm{~h}$ after wounding show the effects of GPC3 silencing on the ability of cells to recover from wounding. The experiments were repeated three times. 

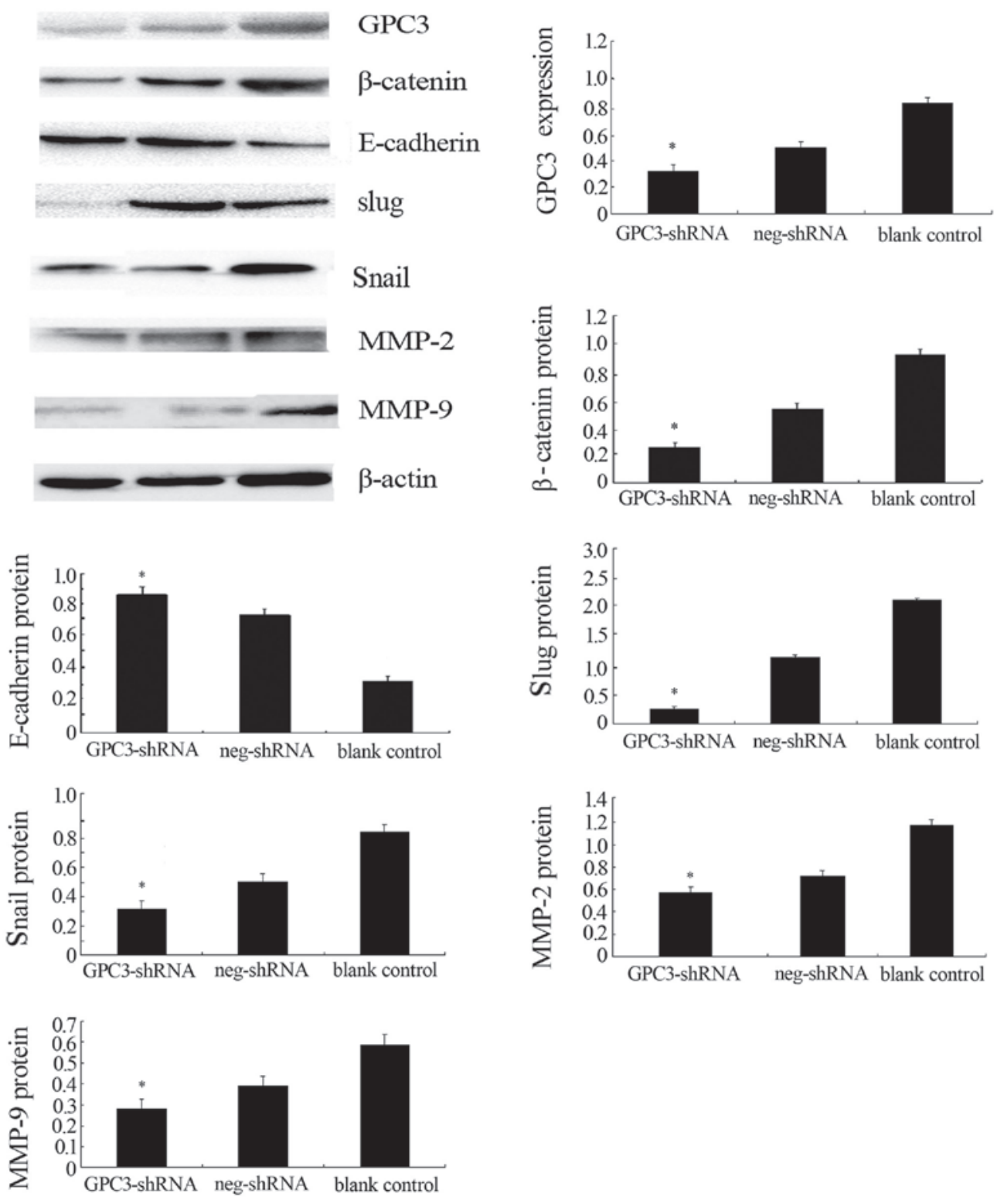

Figure 5. The protein expression of glypican-3 (GPC3), E-cadherin, $\beta$-catenin, Snail, Slug, matrix metalloproteinase-2 (MMP-2), and MMP-9 in HepG2 cells transfected with the GPC3-shRNA was detected by western blotting. At $72 \mathrm{~h}$ after transfection with the GPC3-shRNA, the expression level of GPC3, $\beta$-catenin, Snail, Slug, MMP-2 and MMP-9 is reduced in HepG2 cells; by contrast, the level of E-cadherin is increased. $\beta$-actin was used as an internal normalization control. $\mathrm{P}<0.05$ vs. control groups. The experiments were repeated at least five times.

Alterations in HepG2 cell invasiveness and migration after transfection. The effects of transfection with the GPC3-shRNA on cell invasion were investigated with the Transwell assay. The results of this assay (Fig. 4A) showed that HepG2 cell invasion is considerably inhibited by GPC3 silencing. Forty-eight hours after transfection with the GPC3-shRNA, the number of cells that successfully invaded through the Matrigel was significantly decreased compared to the blank control (Fig. 4B). To further investigate the GPC3-mediated effects on migration, a scratch woundhealing assay was performed. Wound healing was observed and measured at different time-points. At $24 \mathrm{~h}$ after wounding, the blank control cells had covered $>50 \%$ the cell-free area, while the cells transfected with the GPC3-shRNA changed subtly compared with $0 \mathrm{~h}$ after wounding, as shown in Fig. 4C. Overall, these data indicate that the migration rate of GPC3-shRNA-transfected cells was lower than that of the blank control cells at the indicated time-points. We therefore conclude that the migratory and invasive ability of HepG2 cells is tightly linked to the expression of GPC3.

Silencing of GPC3 downregulates the expression of EMT-related proteins in HepG2 cells. To further investigate the mechanism underlying the GPC3 silencing-induced changes in cell invasion and migration, we examined the expression of several invasion-related proteins by western blot analysis. It was previously demonstrated that GPC3 is associated with the Wnt signaling pathway, which is associated with the EMT process $(33,34)$. Here, we examined the expression of the EMT-related proteins E-cadherin, Snail and Slug, of the Wnt signaling-associated protein $\beta$-catenin, and of the migration-related proteins MMP-2 and MMP-9. The results are shown in Fig. 5. The expression of the EMT-related proteins Snail and Slug decreased and that of E-cadherin increased compared to 
the blank and negative controls $(\mathrm{P}<0.05)$. The expression of $\beta$-catenin was markedly decreased after GPC3-shRNA transfection $(\mathrm{P}<0.05)$ and the expression of migrated associated proteins MMP-2 and MMP-9 were decreased in the HCC cells compared with the blank and negative controls $(\mathrm{P}<0.05)$. Therefore, GPC3 had an effect on the migration-associated proteins and the EMT-associated proteins in the HCC cell line. Combined with the data previously mentioned, these results suggested that GPC3 affects the invasion and metastatic abilities in the HCC cell line and had an association with the EMT program, which is important in cell invasion and migration.

\section{Discussion}

The GPC3 protein plays a critical role in HCC oncogenesis. It is associated with cell growth, apoptosis, adhesion and invasion. It may be a valuable diagnostic marker and a potential therapeutic target in HCC. Numerous studies have shown that GPC3 is associated with a number of tumor-related signaling pathways, including Wnt, Hedgehog, SULF, FGF-2, IGF-2, TGF- $\beta$ and BMP-4 (35-44). Among these, the most well studied pathway related to the biological functions of GPC3 is the Wnt pathway. Glypicans are cell surface-anchored heparan sulfate proteoglycans that regulate the activity of Wnts $(45,46)$. GPC3 serves as a selective regulator of Wnt signaling, modulating both the canonical and the non-canonical pathways $(38,39,42)$. It was previously reported that the stimulatory activity of glypicans is based on their ability to act as facilitators of the interaction between Wnts and their receptors (29). The activation of the canonical Wnt signaling pathway has been found to be one of the most frequent events associated with malignant transformation of liver cells (47-49). We found that GPC3-silenced cells exhibit alterations in the Wnt signaling pathway, which is associated with the regulation of cell invasion. We therefore hypothesize that at least in some cell types, GPC3 serves as a selective regulator of Wnt signaling by inhibiting the canonical Wnt signaling pathway. Therefore, GPC3 may stimulate the Wnt/ $\beta$-catenin pathway to induce HCC cell invasion and migration.

Invasion and migration are the main biological characteristics of malignant tumors that cause treatment failure, poor diagnosis and prognosis (3). Therefore, it is of great interest to study the molecular mechanism underlying HCC cell invasiveness. Differentiated epithelial cells can be transformed into mesenchymal cells through a cellular program named epithelial-mesenchymal transition (50). EMT is a key factor in tumor invasion, metastasis and chemotherapy resistance $(51,52)$. It plays a crucial role in local advancement and metastasis of tumors (53). A number of studies have reported that EMT is associated with the invasive and migratory ability of malignant tumors, including esophageal carcinoma, gastric carcinoma, HCC, colorectal cancer and pancreatic cancer (54-59). There are various factors associated with EMT, among which the repression of E-cadherin is an important hallmark of EMT (54). E-cadherin acts as a regulator of cell adhesion in epithelial cells. Downregulation of E-cadherin results in the migration of primary malignant epithelial cells out of their site of origin, where they degrade the surrounding extracellular matrix, migrate into the blood vessels and invade secondary organs (60). Other factors that are involved in the EMT program, such as Snail and Slug, may inhibit the expression of E-cadherin, consequently impairing cell-cell adhesion. The EMT is a complex process that involves crosstalk with several pathways such as TGF, Wnt, PI3K/AKT, Ras-MAPK, Notch, and Hedgehog. Among these pathways, the $\mathrm{Wnt} / \beta$-catenin one plays a crucial role in tumorigenesis $(61,62)$. Recent studies elaborated the role of $\beta$-catenin in cancer metastasis, showing that $\beta$-catenin facilitates EMT in tumor cells (63-64). A proposed mechanism for this involves the complex formed by E-cadherin and $\beta$-catenin; when the E-cadherin level is reduced in the adherens junctions, its partner $\beta$-catenin is released into the cytosol, where it can activate LEF/TCF-mediated transcription and drive the expression of important cell-cycle proteins and oncogenes; via this mechanism, $\beta$-catenin signaling may contribute to EMT and eventually result in tumor invasion and metastasis (65). On the other hand, several studies also suggested that $\beta$-cateninmediated transcription can induce the expression of Slug and Snail, thereby contributing to the EMT program (66-67). Extensive studies in various developmental EMT systems provide convincing evidence that Wnt signaling is a key event of EMT $(33,34,51,57,58,63,68)$, although the precise signaling pathway activated by individual family members may differ among EMT events in different systems.

In this study, we hypothesized that silencing of $G P C 3$ may decrease HCC cell invasion and migration, and provided experimental evidence supporting this hypothesis. EMT-related proteins were detected in HCC cells by western blot analysis, and the invasive ability of these cell lines was assessed following transfection using the Matrigel Transwell and the wound healing assays. Furthermore, the expression of certain critical proteins in the $\mathrm{Wnt} / \beta$-catenin signaling pathway was also analyzed by western blot analysis. In this study, the main focus was the potential relationship between EMT, $\beta$-catenin and GPC3. Our findings demonstrated that cell invasion and migration are inhibited following transfection (Figs. 4 and 5). Silencing of GPC3 reduced the protein level of Snail and Slug and increased the level of E-cadherin, potentially contributing in inhibition of the EMT program, which also inhibited the $\mathrm{HCC}$ invasive and metastatic activities, in agreement with the reduced expression of $\beta$-catenin. Based on the marked cellular changes observed in our study, we propose that the cell invasive ability that is associated with the EMT process in HCC may be regulated, at least in part, by the expression of GPC3.

In summary, this study demonstrated that GPC3 induces HCC invasiveness and metastasis, potentially via induction of EMT. These data are novel and important for the understanding of the role of GPC3 in HCC and the future development of gene therapy for this type of tumor. However, further studies are needed to elucidate the relationship between GPC3-mediated cell invasion and the EMT program.

\section{Acknowledgements}

This study was supported by the Project of Science and Technology of Liaoning (2011415052-3).

\section{References}

1. Mazzanti R, Gramantieri L and Bolondi L: Hepatocellular carcinoma: epidemiology and clinical aspects. Mol Aspects Med 29: 130-143, 2008. 
2. Kawano Y, Sasaki A, Kai S, et al: Short- and long-term outcomes after hepatic resection for hepatocellular carcinoma with concomitant esophageal varices in patients with cirrhosis. Ann Surg Oncol 15: 1670-1676, 2008

3. Poon RT, Fan ST and Wong J: Risk factors, prevention and management of postoperative recurrence after resection of hepatocellular carcinoma. Ann Surg 232: 10-24, 2000.

4. Mischak H, Allmaier G, Apweiler R, et al: Recommendations for biomarker identification and qualification in clinical proteomics. Sci Transl Med 2: 42-46, 2010

5. Mann C D, Neal C P, Garcea G, et al: Prognostic molecular markers in hepatocellular carcinoma: a systematic review. Eur J Cancer 43: 979-992, 2007

6. Wang Y, Shen Z, Zhu Z, et al: Clinical values of AFP, GPC3 mRNA in peripheral blood for prediction of hepatocellular carcinoma recurrence following OLT:AFP,GPC3 mRNA for prediction of HCC.Hepat Mon 11: 195-199, 2011

7. Huynh H: Molecularly targeted therapy in hepatocellular carcinoma. Biochem Pharmacol 80: 550-560, 2010.

8. Marra M, Sordelli I M, Lombardi A, et al: Molecular targets and oxidative stress biomarkers in hepatocellular carcinoma: an overview. J Transl Med 9: 171-185, 2011

9. Gonzalez SA, Keeffe EB: Diagnosis of hepatocellular carcinoma: role of tumor markers and liver biopsy. Clin Liver Dis 15 : 297-306, 2011

10. Zou ZQ, Ding YP, Long B, et al: Gpc-3 is a notable diagnostic, prognostic and a latent targeted therapy marker in hepatocellular carcinoma. Hepatogastroenterology 57: 1285-1290, 2010.

11. Capurro M, Wanless IR, Sherman M, et al: Glypican-3: a novel serum and histochemical marker for hepatocellular carcinoma. Gastroenterology 125: 89-97, 2003.

12. Yamauchi N, Watanabe A, Hishinuma $\mathrm{M}$, et al: The glypican 3 oncofetal protein is a promising diagnostic marker for hepatocellular carcinoma. Mod Pathol 18: 1591-1598, 2005.

13. Libbrecht L, Severi T, Cassiman D, et al: Glypican-3 expression distinguishes small hepatocellular carcinomas from cirrhosis, dysplastic nodules, and focal nodular hyperplasia-like nodules Am J Surg Pathol 30: 1405-1411, 2006.

14. Liovet JM, Chen Y, Wurmbach E, et al: A molecular signature to discriminate dysplastic nodules from early hepatocellula carcinoma in HCV-cirrhosis. Gastroenterology 131: 1758-1767, 2006.

15. Nakatsura T, Kageshita T, Ito S, et al: Identification of glypican-3 as a novel tumor marker for melanoma. Clin Cancer Res 10: 6612-6621, 2004.

16. Stadlmann S, Gueth U, Baumhoer D, et al: Glypican-3 expression in primary and recurrent ovarian carcinomas. Int J Gynecol Pathol 26: 341-344, 2007.

17. Zynger DL, Dimov ND, Luan C, et al: Glypican 3: a novel marker in testicular germ cell tumors. Am J Surg Pathol 30: 1570-1575, 2006.

18. Baumhoer D, Tornillo L, Stadlmann S, et al: Glypican 3 expression in human nonneoplastic, preneoplastic, and neoplastic tissues: a tissue microarray analysis of 4,387 tissue samples. Am J Clin Pathol 129: 899-906, 2008.

19. Saikali Z and Sinnett D: Expression of glypican 3 (GPC3) in embryonal tumors. Int J Cancer 89: 418-422, 2000.

20. Kim H, Xu GL, Borczuk AC, et al: The heparan sulfate proteoglycan GPC3 is a potential lung tumor suppressor. Am J Respir Cell Mol Biol 29: 694-701, 2003.

21. Murthy SS, Shen T, De Rienzo A, et al: Expression of GPC3, an X-linked recessive overgrowth gene, is silenced in malignant mesothelioma. Oncogene 19: 410-416, 2000.

22. Xiang YY, Ladeda V and Filmus J: Glypican-3 expression is silenced in human breast cancer. Oncogene 20: 7408-7412, 2001

23. Zhu ZW, Friess H, Wang L, et al: Enhanced glypican-3 expression differentiates the majority of hepatocellular carcinomas from benign hepatic disorders. Gut 48: 558-564, 2001.

24. Filmus J and Capurro M: The role of glypican-3 in the regulation of body size and cancer. Cell Cycle 7: 2787-2790, 2008

25. Stigliano I, Puricelli L, Filmus J, et al: Glypican-3 regulates migration, adhesion and actin cytoskeleton organization in mammary tumor cells through Wnt signaling modulation. Breast Cancer Res Treat 114: 251-262, 2009.

26. Filmus J, Church JG and Buick RN: Isolation of a cDNA corresponding to a developmentally regulated transcript in rat intestine. Mol Cell Biol 8: 4243-4249, 1998.

27. Pilia G, Hughes-Benzie RM, MacKenzie A, et al: Mutations in GPC3, a glypican gene, cause the Simpson-Golabi-Behmel overgrowth syndrome. Nat Genet 12: 241-247, 1996.
28. De Cat B and David G: Developmental roles of the glypicans Semin Cell Dev Biol 12: 117-125, 2001.

29. Capurro MI, Xiang YY, Lobe C and Filmus J: Glypican-3 promotes the growth of hepatocellular carcinoma by stimulating canonical Wnt signaling. Cancer Res 65: 6245-6254, 2005.

30. Ruan J, Liu F, Chen X, et al: Inhibition of glypican-3 expression via RNA interference influences the growth and invasive ability of the MHCC97-H human hepatocellular carcinoma cell line. International journal of molecular medicine 28: 497-503, 2011.

31. Yang J, Mani SA, Donaher JL, et al: Twist, a master regulator of morphogenesis, plays an essential role in tumor metastasis. Cell 117: 927-939, 2004

32. Livak KJ and Schmittgen TD: Analysis of relative gene expression data using real-time quantitative PCR and the $2(\mathrm{~T})$ (-Delta Delta C) method. Methods 25:402-408,2001.

33. Howard S, Deroo T, Fujita Y, et al: A positive role of cadherin in Wnt/ $\beta$-catenin signalling during epithelial-mesenchymal transition. PloS one 6: e23899,2011

34. Yook J I, Li X Y, Ota I, et al: Wnt-dependent regulation of the E-cadherin repressor snail. Journal of Biological Chemistry 280 $11740-11748,2005$

35. Baeg GH and Perrimon N: Functional binding of secreted molecules to heparan sulfate proteoglycans in Drosophila. Curr Opin Cell Biol 12: 575-580, 2000.

36. Jackson SM, Nakato H, Sugiura M, et al: dally, a Drosophila glypican, controls cellular responses to the TGF- $\beta$-related morphogen Dpp. Development 124: 4113-4120, 1997.

37. Perrimon $\mathrm{N}$ and Bernfield M: Specificities of heparin sulphate proteoglycans in developmental processes. Nature 404: 725-728, 2000.

38. Topczewsky J, Sepich DS, Myers DC, et al: The zebrafish glypican knypek controls cell polarity during gastrulation movements of convergent extension. Dev Cell 1: 251-264, 2001

39. Ohkarawa B, Yamamoto TS, Tada M and Ueno N: Role of glypican 4 in the regulation of convergent extension movements during gastrulation in Xenopus laevis. Development 130: 2129-2138, 2003.

40. De Cat B, Muyldermans SY, Coomans C, et al: Processing by proprotein convertases is required for glypican-3 modulation of cell survival, Wnt signaling, and gastrulation movements. J Cell Biol 163: 625-635, 2003.

41. Kramer KL and Yost HJ: Heparan sulfate core proteins in cell-cell signaling. Annu Rev Genet 37: 461-484, 2003.

42. Baeg GH, Lin X, Khare N, et al: Heparan sulfate proteoglycans are critical for the organization of the extracellular distribution of Wingless. Development 128: 87-94, 2001.

43. Han C, Belenkaya TY, Wang B and Lin X: Drosophila glypicans control the cell-to-cell movement of hedgehog by a dynamin-independent process. Development 131: 601-611, 2004.

44. Lum L, Yao S, Mozer B, et al: Identification of Hedgehog pathway components by RNAi in Drosophila cultured cells. Science 299: 2039-2045, 2003

45. Filmus J, Capurro M and Rast J: Glypicans. Genome Biol 9: 224 , 2008.

46. Song HH and Filmus J: The role of glypicans in mammalian development. Biochim Biophys Acta 1573: 241-246, 2002.

47. Feitelson MA, Sun B, Satiroglu Tufan NL, et al: Genetic mechanisms of hepatocarcinogenesis. Oncogene 21: 2593-2604, 2002.

48. Kern MA, Breuhahn K and Schirmacher P: Molecular pathogenesis of human hepatocellular carcinoma. Adv Cancer Res 86 67-112, 2002.

49. Satoh S, Daigo Y, Furukawa Y, et al: AXIN1 mutations in hepatocellular carcinomas, and growth suppression in cancer cells by virus-mediated transfer of AXIN1. Nat Genet 24: 245-250, 2000

50. Yang J and Weinberg RA: Epithelial-mesenchymal transition: at the crossroads of development and tumor metastasis. Dey Cell 14: 818-829, 2008.

51. Li X, Xu Y, Chen Y, et al: SOX2 promotes tumor metastasis by stimulating epithelial-to-mesenchymal transition via regulation of WNT/ß-catenin signal network. Cancer Lett 336: 379-389, 2013.

52. van Zijl F, Zulehner G, Petz M, et al: Epithelial-mesenchymal transition in hepatocellular carcinoma. Future Oncol 5: 1169-1179, 2009.

53. Weinberg RA: Mechanisms of malignant progression. Carcinogenesis 29: 1092-1095, 2008

54. Thiery JP: Epithelial-mesenchymal transitions in development and pathologies. Curr Opin Cell Biol 15: 740-746, 2003.

55. Thompson EW and Newgreen DF: Carcinoma invasion and metastasis: a role for epithelial-mesenchymal transition? Cancer Res 65: 5991-5995, 2005 
56. Vincan E, Barker N: The upstream components of the Wnt signalling pathway in the dynamic EMT and MET associated with colorectal cancer progression. Clinical Exp Metastasis 25: 657-663, 2008.

57. Prasad C P, Mirza S, Sharma G, et al: Epigenetic alterations of $\mathrm{CDH} 1$ and APC genes: Relationship with activation of Wnt $/ \beta$ catenin Pathway in invasive ductal carcinoma of breast. Life Sci 83: 318-325, 2008

58. Zhao J H, Luo Y, Jiang Y G, et al: Knockdown of $\beta$-Catenin through shRNA cause a reversal of EMT and metastatic phenotypes induced by HIF-1 $\alpha$. Cancer Invest 29: 377-382, 2011.

59. Miyoshi A, Kitajima Y, Kido S, et al: Snail accelerates cancer invasion by upregulating MMP expression and is associated with poor prognosis of hepatocellular carcinoma. $\mathrm{Br}$ J Cancer 92:252-258, 2005.

60. Liotta LA: Tumor invasion and metastases - role of the extracellular matrix: Rhoads Memorial Award lecture. Cancer Res 46: 1-7, 1986.

61. Ban KC, Singh H, Krishnan R and Seow HF: GSK-3beta phosphorylation and alteration of beta-catenin in hepatocellular carcinoma. Cancer Lett 199: 201-208, 2003.

62. Lucero OM, Dawson DW, Moon RT and Chien AJ: A reevaluation of the 'oncogenic' nature of Wnt/beta-catenin signaling in melanoma and other cancers. Curr Oncol Rep 12: 314-318, 2010.
63. Sánchez-Tilló E, de Barrios O, Siles L, et al: $\beta$-catenin/TCF4 complex induces the epithelial-to-mesenchymal transition (EMT)-activator ZEB1 to regulate tumor invasiveness. Proc Natl Acad Sci USA 108: 19204-19209, 2011.

64. Schmalhofer O, Brabletz S and Brabletz T: E-cadherin, $\beta$-catenin, and ZEB1 in malignant progression of cancer. Cancer Metastasis Rev 28: 151-166, 2009.

65. Kim K, Daniels KJ and Hay ED: Tissue-specific expression of beta-catenin in normal mesenchyme and uveal melanomas and its effect on invasiveness. Exp Cell Res 245: 79-90, 1998.

66. Conacci-Sorrell M,Simcha I, Ben-Yedidia T, et al: Autoregulation of E-cadherin expression by cadherin-cadherin interactions: the roles of beta-catenin signaling, Slug, and MAPK. J Cell Biol 163: 847-857, 2003.

67. Onder TT, Gupta PB, Mani S, et al: Loss of E-cadherin promotes metastasis via multiple downstream transcriptional pathways Cancer Res 68: 3645-3654, 2008.

68. Oh SJ, Shin JH, Kim TH, et al: $\beta$-Catenin activation contributes to the pathogenesis of adenomyosis through epithelial-mesenchymal transition. J Path 231: 210-222, 2013. 